DOE/ER/13065 - T3

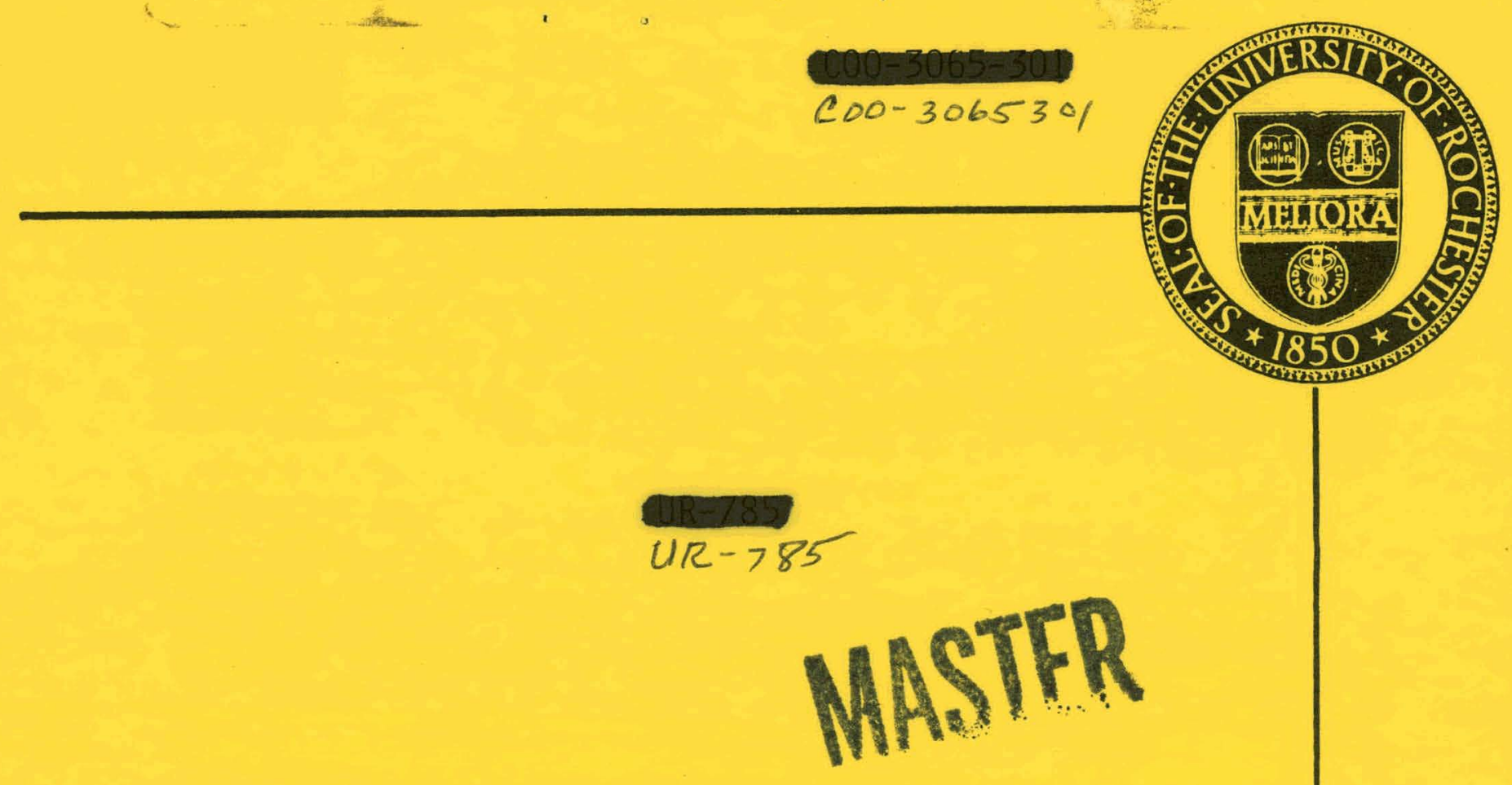

Decay Constants of Pseudoscalar Mesons Containing Heavy Quarks

by

V. S. Mathur and Mieko T, Yamawaki

THE UNIVERSITY OF ROCHESTER

DEPARTMENT OF PHYSICS AND ASTRONOMY

ROCHESTER， NEW YORK

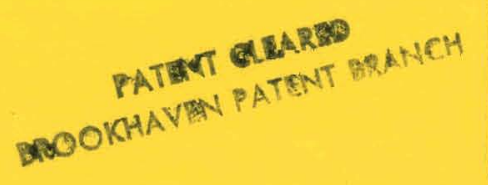

DISTRIBUTION OF THIS DOCUMENT IS UNLIMITED 


\section{DISCLAIMER}

This report was prepared as an account of work sponsored by an agency of the United States Government. Neither the United States Government nor any agency Thereof, nor any of their employees, makes any warranty, express or implied, or assumes any legal liability or responsibility for the accuracy, completeness, or usefulness of any information, apparatus, product, or process disclosed, or represents that its use would not infringe privately owned rights. Reference herein to any specific commercial product, process, or service by trade name, trademark, manufacturer, or otherwise does not necessarily constitute or imply its endorsement, recommendation, or favoring by the United States Government or any agency thereof. The views and opinions of authors expressed herein do not necessarily state or reflect those of the United States Government or any agency thereof. 


\section{DISCLAIMER}

Portions of this document may be illegible in electronic image products. Images are produced from the best available original document. 


\title{
Decay Constants of Pseudoscalar Mesons Containing \\ Heavy Quarks
}

\author{
V. S. Mathur and Mieko T. Yamawaki \\ Department of Physics and Astronomy \\ University of Rorhester \\ Rochester; N. Y. 14627
}

\section{Abstract}

The QCD sum-rules of Shifman et al. for $n^{\text {th }}$ order moments are applied to the determination of the decay constants of pseudoscalar mesons containing a heavy quark ( $c$ or b). We consider the general case when $Q^{2}$, the squared momentum transfer, is non-zero. We discuss the stability of the sum-rules against variations in both $Q^{2}$ and $n$.

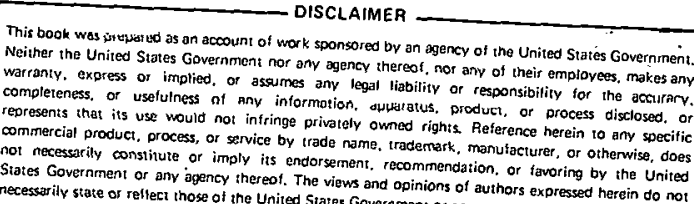


The decay constants of heavy mesons have played an important role in several recent calculations such as the non-spectator contribution to charmed meson decays ${ }^{2}$, the estimation of the branching ratios ${ }^{2}$ of decay modes like $F \rightarrow \tau \bar{\nu}_{\tau}, B \rightarrow \tau \bar{\nu}_{\tau}$, etc. Accordingly, they have been the subject of much investigation recently and many different techniques have been used in their estimation ${ }^{3-5}$. Unfortunately, these methods have led to a wide spectrum of values.

Recently, a promising approach has been developed by Shifman et al. 6 who have found a way to apply Quantum Chromodynamics (QCD) to resonance physics. Here, starting with the polarization tensor of suitable currents, one writes, on the one hand, a dispersion represenation for it, and on the other, the operator product expansion (OPE). Usually OPE is valid for large momentum transfers, but shifman et al. have argued that for heavy quarks the shori aistance expansion can be valid even at zero momentum tranfers. For the current $J_{\mu}$, defining

$$
\begin{aligned}
\pi_{\mu \nu}(q) & =i \int d^{4} x e^{i q \cdot x}\left\langle 0\left|T^{*}\left(J_{\mu}(x) J_{\nu}(0)\right)\right| 0\right\rangle \\
& =-g_{\mu \nu} \pi_{I}\left(Q^{2}\right)+q_{\mu} q_{\nu} \pi_{2}\left(Q^{2}\right), Q^{2}=-q^{2}
\end{aligned}
$$

one can then write down expansions for the polarization functions $\pi_{1,2}$ utilizing OPE. For currents involving heavy quarks, sum-rules for moments of the polarization functions

$$
\left.\frac{1}{n !}\left(-\frac{\partial}{\partial Q^{2}}\right)^{n} \pi\left(Q^{2}\right)\right|_{Q^{2}=0}=\frac{1}{\pi} \int \frac{\operatorname{Im} \pi(s)}{s^{n+1}} d s
$$

can then be derived at $\mathrm{Q}^{2}=0$. Using perturbative $\mathrm{QCD}$ in the calculation of the coefficient functions in OPE, and saturating the disperison integral by low lying states, Shifman et al. ${ }^{4,6,7}$ have obtained very interesting results with appropriate choices of $n$. 
Recently Reinders et al. ${ }^{8}$ have extended this technique by considering $Q^{2} \neq 0$, and have successfully calculated the charmomium spectrum. In this paper, we calculate the pseudoscalar decay constants of D, F and the B-mesons using a similar approach. We also discuss the choice of $\mathrm{n}$ and $Q^{2}$ from considerations of the stability of the sum-rules.

For axial vector currents involving a heavy quark, utilizing OPE, we obtain from Eq. (1)

$$
\begin{aligned}
\pi_{\mu \nu}(q)= & \left.c_{\mu \nu}^{I}(q)+c_{\mu \nu}^{G}(q)<0\left|G_{\alpha \beta}^{a} G_{\alpha \beta}^{a}\right| 0\right\rangle \\
& \left.+c_{\mu \nu}^{M}(q)<0|\bar{\psi} M \psi| 0\right\rangle+\ldots \ldots
\end{aligned}
$$

where the notation is the same as in Ref. (6). In perturbative QCD only the $c_{\mu \nu}^{I}(q)$ term, arising from the unit operator in the expansion, survives. Although Shifman et al. have advocated that the vacuum expectation values of higher dimensional operators need not vanish as a consequence of the non-perturbative structure of the vacuum, we will in this paper drop all terms on RHS of Eq. (3) except the first, because the successive terms in the series are suppressed by higher and higher powers of the heavy quark mass. Futhermore, since asymptotic freedom is realized with heavy quarks even for low values of the momentum transfer, we calculate $C_{\mu \nu}^{I}(q)$ using $Q C D$ only in the lowest order. Writing $\pi_{\mu \nu}$ in terms of its longitudinal and transverse components

$$
\pi_{\mu \nu}(q)=\left(\frac{q_{\mu} q_{\nu}}{q^{2}}-g_{\mu \nu}\right) \pi_{t}\left(Q^{2}\right)+\frac{q_{\mu} q_{\nu}}{q^{2}} \pi_{\ell}\left(Q^{2}\right)
$$

standard lowest order QCD calculations then determine $\pi_{t}$ and $\pi_{\ell}$. In our work here, we are interested in $\pi_{l}$ which gets contributions only from pseudoscalar states. If $M$ and $m$ are the masses of the heavy and light quarks, we obtain 


$$
\begin{array}{r}
\pi_{\ell}\left(Q^{2}\right)=-\frac{3}{4 \pi^{2}} \int_{0}^{1} d x\left[M+m^{2} x+M^{2}(1-x)\right] \\
\\
\quad \ln \left[1+\frac{Q^{2} x(1-x)}{m^{2} x+M^{2}(1-x)}\right]
\end{array}
$$

We now consider the $n^{\text {th }}$ moment of $\pi_{\hat{i}}\left(Q^{2}\right)$ for arbitrary $Q^{2}$, using a dispersion representation for $\pi_{\ell}$ with $\leq n$ subtractions.

$$
M_{n} \equiv \frac{1}{n !}\left(-\frac{\partial}{\partial Q^{2}}\right)^{n} \pi_{\ell}\left(Q^{2}\right)=\frac{1}{\pi} \int \frac{\operatorname{Im} \pi_{\ell}(s)}{\left(s+Q^{2}\right)^{n+1}} d s
$$

Separating out the lowest lying pseudoscalar meson contribution, we obtain

$$
M_{n}=\frac{f_{p}^{2} M_{p}^{2}}{\left(M_{p}^{2}+Q^{2}\right)^{n+1}}+\text { continuum }
$$

where $M_{p}$ is the mass of the heavy pseudoscalar meson and $f_{p}$ its decay constant. Dropping the continuum contribution and calculating the $n^{\text {th }}$ moment of $\pi_{\ell}$ from Eq. (5), we get the desired relation

$$
\frac{f_{p}^{2} M_{p}^{2}}{\left(M_{p}^{2}+Q^{2}\right)^{n+1}}=\frac{3}{4 \pi^{2}} \frac{1}{n} \int_{0}^{1} d x \frac{x^{n}(1-x)^{n}\left[M+m^{2} x+M^{2}(1-x)\right]}{\left[m^{2} x+M^{2}(1-x)+Q^{2} x(1-x)\right]^{n}}
$$

Eq. (8) can determine the decay constant $f_{p}$ if we know the quark masses and $M_{p}$. However, an important question is what do we use for $n$ and $Q^{2}$ ? To investigate this question, let us consider the restrictions on $\mathrm{n}$ and $\mathrm{Q}^{2}$ imposed by the requirement that the terms neglected in obtaining the sum-rule (8) are small compared with the terms we have retained. Regarding the continuum corrections, simple models like replacing the continuum by effective poles or approximating the continuum by parton model, show that such corrections are small compared with the contribution of the lowest lying pseudoscalar meson for large $n$ and small $\mathrm{Q}^{2}$. On the other hand, the neglect of higher order $\mathrm{QCD}$ corrections and the non-perturbative terms 
in the RHS of Eq. (8), is more justified for small ${ }^{6} \mathrm{n}$ and large $Q^{2}$. Clearly, the validity of Eq. (8) rests on the possibility of finding a compromise choice for $\mathrm{n}$ and $\mathrm{Q}^{2}$. In practice whether such a choice exists and what it is, can be studied by first calculating $f_{p}$ from Eq. (8) for a wide set of values of $n$ and $Q^{2}$. Now, if there exists a domain for $n$ and $Q^{2}$ where $f_{p}$ is sensibly a constant, so that the sum-rule is reasonably stable, we have found the compromise choice.

In Fig. 1, we have plotted $f_{D}$ for several values of $n$ and $Q^{2}$. In computing $\mathrm{E}_{\mathrm{D}}$ we have used the masses $\mathrm{M}_{\mathrm{D}}=1.86 \mathrm{GeV}, \mathrm{m}_{\mathrm{u}} \simeq \mathrm{m}_{\mathrm{d}} \simeq 0.01 \mathrm{GeV}$ and $M_{c}=1.27 \mathrm{Gev} .9$ To illustrate our arguments described above, consider Fig. 1(a). Clearly for $n=3$ or $4, f_{p}$ is reasonably flat with respect to variations in $\sqrt{Q^{2}}$ over the range shown. Similarly Fig. I(b) shows that for $\sqrt{Q^{2}} \sim 0.8 \mathrm{GeV}, \mathrm{f}_{\mathrm{p}}$ is sensibly constant for a range of values of $n$. We thus find that the sum-rule (8) works best around $n=3-4, \sqrt{Q^{2}} \sim 0.8 \mathrm{GeV}$. To pinpoint the 'best' values of $\mathrm{n}$ and $\sqrt{Q^{2}}$, all one has to do is to look at the value of $\sqrt{Q^{2}}$ in Fig. $I(a)$ where the dispersion in $f_{p}$ for different $\mathrm{n}$ values is the least, and similarly to look at the value of $\mathrm{n}$ in Fig. $\mathrm{I(b)}$ where the $f_{D}$-curves for different $\sqrt{Q^{2}}$ values converge the most. This fixes the 'best' values at $n=3, \sqrt{Q^{2}}=0.9 \mathrm{GeV}$ for which we get $f_{D} \simeq 144 \mathrm{MeV}$. In Figs. 2-5 we do the same analysis for the other $f_{p}$ 's where $P=F$, $\mathrm{B}_{\mathrm{u}}, \mathrm{B}_{\mathrm{S}}$ and $\mathrm{B}_{\mathrm{C}^{\prime}}$ respectively. The masses of some of these mesons are not. known, but for our purposes here we choose them to be $M_{F}=2.03 \mathrm{GeV}, 10$ $\mathrm{M}_{\mathrm{B}_{\mathrm{u}}} \simeq 5.2 \mathrm{GeV},{ }^{11} \mathrm{M}_{\mathrm{B}_{\mathrm{S}}} \simeq 5.5 \mathrm{GeV}$ and $\mathrm{M}_{\mathrm{B}_{C}} \simeq 6.6 \mathrm{GeV}$. The mass of $\mathrm{s}$ and $\mathrm{b}$ quarks are taken as $0.15 \mathrm{GeV}$ and $4.24 \mathrm{GeV}^{9}$ respectively. We display the results for the 'best' values of $n$ and $\sqrt{Q^{2}}$ in each case with the corresponding values of $f_{p}$ in the table. Our results for $f_{D}$ and $f_{F}$ are not very different. from those obtained from the $Q^{2}=0$ sum-rules, ${ }^{4}$ but the 
stability considerations discussed here for arbitrary $\mathbf{n}$ and $\mathbf{Q}^{2}$ provide extra reliability.

With the calculated values of $\mathrm{f}_{\mathrm{p}}$, we can estimate the $\tau$-leptonic decay rates of the mesons $F, B_{u}$ and $B_{c}$. For $P \rightarrow \tau \bar{\nu}_{\tau}$, the decay rate is given by

$$
\Gamma\left(P \rightarrow \tau \bar{\nu}_{\tau}\right)=\left.\left.\frac{1}{8 \pi}\right|_{Q q}\right|^{2} G_{F}^{2} f_{p}^{2} M_{p} m_{\tau}^{2}\left(1-m_{\tau / M}^{2}\right)
$$

where $U_{Q q}$ is an element of the Kobayashi-Maskawa mixing matrix. ${ }^{12}$ Using the $M_{p}$ 's quoted above and $f_{p}$ from the table, we get

$$
\begin{aligned}
& \Gamma\left(F \rightarrow \tau \bar{v}_{\tau}\right)=1.25 \times 10^{11}\left|\mathrm{u}_{\mathrm{cs}}\right|^{2} \mathrm{~s}^{-1} \\
& \Gamma\left(B_{\mathrm{u}} \rightarrow \tau \bar{v}_{\tau}\right)=4.88 \times 10^{12}\left|\mathrm{U}_{\mathrm{bu}}\right|^{2} \mathrm{~s}^{-1} \\
& \Gamma\left(B_{\mathrm{c}} \rightarrow \tau \bar{v}_{\tau}\right)=5.16 \times 10^{13} /\left.\mathrm{U}_{\mathrm{bc}}\right|^{2} \mathrm{~s}^{-1}
\end{aligned}
$$

We may now use the favored values ${ }^{2}$ of $\mathrm{U}_{\mathrm{Qq}}:\left|\mathrm{u}_{\mathrm{cs}}\right|=0.97,\left|\mathrm{u}_{\mathrm{bu}}\right|=0.07-0.12$, and $\left|U_{b c}\right|=0.13-0.16$. The lifetime of $F-m e s o n^{10}$ is known with large uncertainties $\tau(F)=2.2+2.8 \times 10^{-13} \mathrm{~s}$; for $B$-mesons, we may use the theoretical estimates $^{2,13}: \tau\left(B_{u}\right) \sim(5-10) \times 10^{-14} \mathrm{~s}$ and $\tau\left(B_{c}\right) \sim(1-3) \times 10^{-14} \mathrm{~s}$. Using these values in Eq. (10), we predict the branching ratios

$$
\begin{aligned}
& \mathrm{BR}\left(F \rightarrow \tau \bar{\nu}_{\tau}\right)=2.6_{-1.2}^{+3.3} \% \\
& \mathrm{BR}\left(\mathrm{B}_{\mathrm{u}} \rightarrow \tau \bar{\nu}_{\tau}\right)=(0.12-0.7) \% \\
& \mathrm{BR}\left(\mathrm{B}_{\mathrm{C}} \rightarrow \tau \bar{\nu}_{\tau}\right)=(0.9-4) \%
\end{aligned}
$$

We would like to thank J. Chakrabarti and I. P. S. Sirgh for many useful discussions.

This work was supported by the U.S. Department of Energy under Contract No. DE-AC02-76ER13065. 
TABLE

\begin{tabular}{|c|c|c|c|}
\hline$P$ & 'best' $n$ & 'best' $\sqrt{Q^{2}}(\mathrm{Gev})$ & $f_{p}($ Mev $)$ \\
\hline D & 3 & 0.9 & 144 \\
\hline $\mathbf{F}$ & 2 & 0.8. & 214 \\
\hline${ }^{B} \mathrm{u}$ & 7 & $2: 0$ & 216 \\
\hline${ }^{B} \cdot S$ & 5 & 2.0 & 319 \\
\hline$B_{C}$ & 4 & 1.6 & 593 \\
\hline
\end{tabular}


Table Caption

Calculated results for $f_{p}$ for the 'best' values of $n$ and $\sqrt{Q^{2}}$ obtained from the Figs. 1-5.

Figure Captions

(a) $f_{p}$ vs $\sqrt{Q^{2}}$ for a few selected values of $n$.

(b) $f_{p}$ vs $n$ for different $\sqrt{Q^{2}}$. 
$\underline{\text { References }}$

1. M. Bander, D. Silverman, and A. Soni, Phys. Rev. Lett. 44,7 (1980).

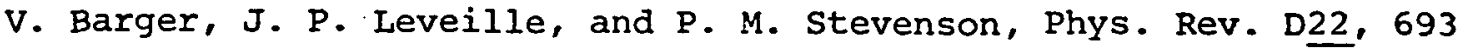
(1980) .

2. V. Barger, J. P. Leveille, P. M. Stevenson, and R. J. N. Phillips, Phys. Rev. Lett. $\underline{45}, 83$ (1980).

3. N. G. Deshpande and D. Iskander, Phys. Rev. D19, 3457 (1979) and references therein.

S. S. Gershtein and N. Yu. Khlopov Pis'ma Zh. Eksp. Teor. Fiz 23, 374. (1976) [JETP Lett. $23 ; 338$ (1976)]

4. V. A. Novikov, L. B. Okun, M. A. Shifman, A. I. Vainshtein, M. B. Voloshin, and V. I. Zakharov, Phys. Rev. Lett. 38, 626 (1977).

5. M. Suzuki, Nucl. Phys. B 177, 413 (1980).

6. M. A. Sinimman, A. I. Vainshtein, and V. I. Zakharov, Nucl. Phys. B $1.47,385,448$ (1979).

V. A. Novikov, I. B. Okun, M. A. Shifman, A. I. Vainshtein, M. B. Voloshin, and V. I. Zakharov, Phys. Report 41, No. 1 (1978).

7. M. A. Shifman, A. I. Vainshtein, M. B. Voloshir and V. I. Zakharov, Phys . Lett. 77B, 80 (1978).

8. L. J. Reinders, H. R. Rubinstein and S. Yazaki, Phys. Lett. 95B, 103 (1980).

9. We have calculated the masses of the $c$ and $b$ quarks by using the lowest order sum-rules in Ref. 6 for $Q^{2} \neq 0$, and the masses and $e^{-} e^{+}$ decay widths of the lowest four resonances of the $\psi$ and the $\gamma$ families, respectively. strictly speaking, the quark mass depends on $Q^{2}$, and is usually defined at some suitable Euclidean point. However, the difference between various renormalization points becomes 
apparent only if higher order QCD terms are included.

10. K. Niu, Proceedings of the XX conference in High Energy Physics, American Institute of Physics, New York 1981.

N. Ushida et al., Phys. Rev. Lett. 45, 1053 (1980).

11. D. Andrews et al., Phys. Rev. Lett. 45, 219 (1980).

G. Finocchiaro et al., Phys. Rev. Lett. $\underline{45}, 222$ (1980).

12. M. Kobayashi and T. Maskawa, Prog. Theor. Phys. 49, 652 (1973).

13. K. Jagannathan, Ph.D. thesis, University of Rochester, 1980. 
Fig $1-(a)$
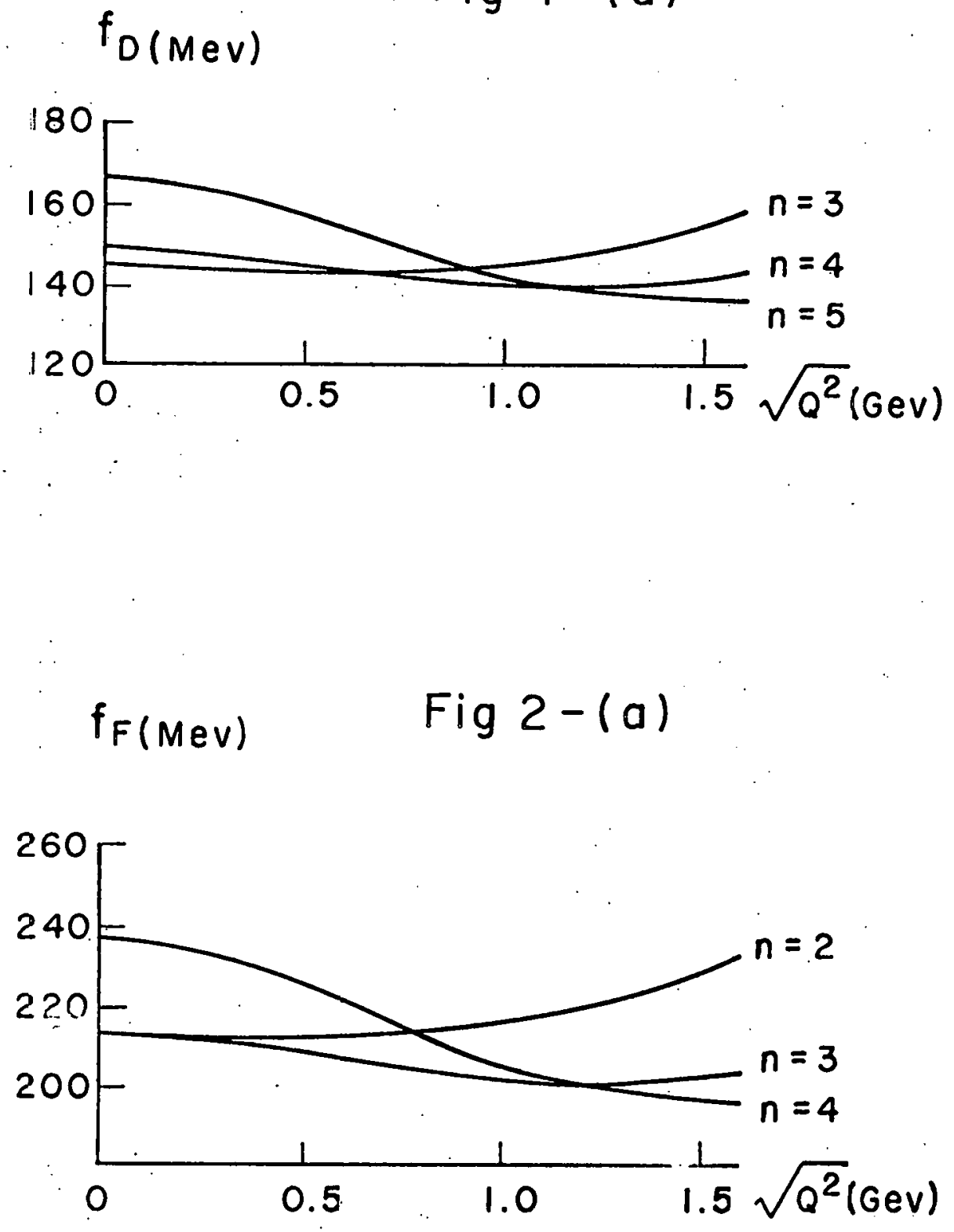

Fig $1-(b)$
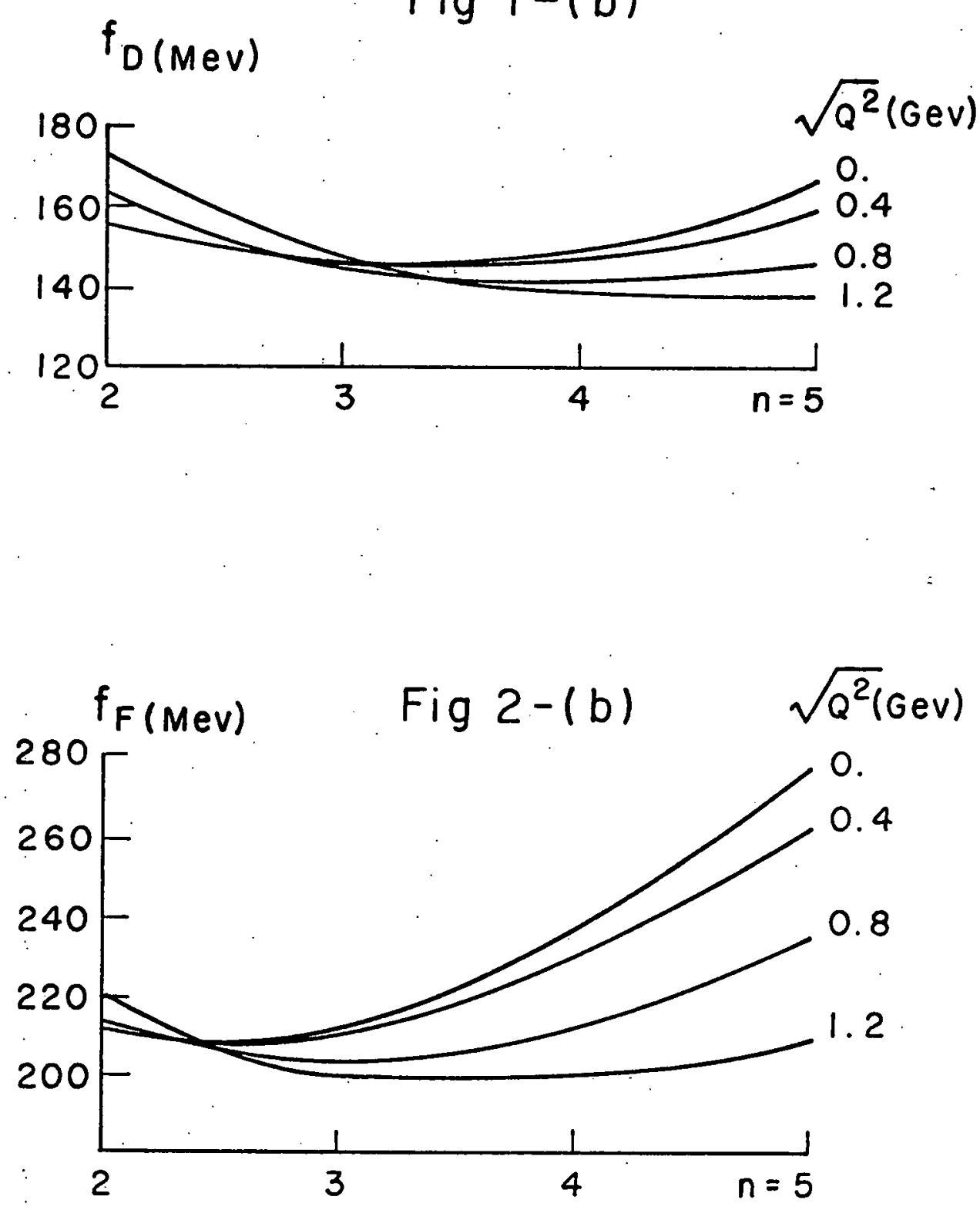
Fig $3-(a)$

$$
f_{B u} \text { (Mev) }
$$

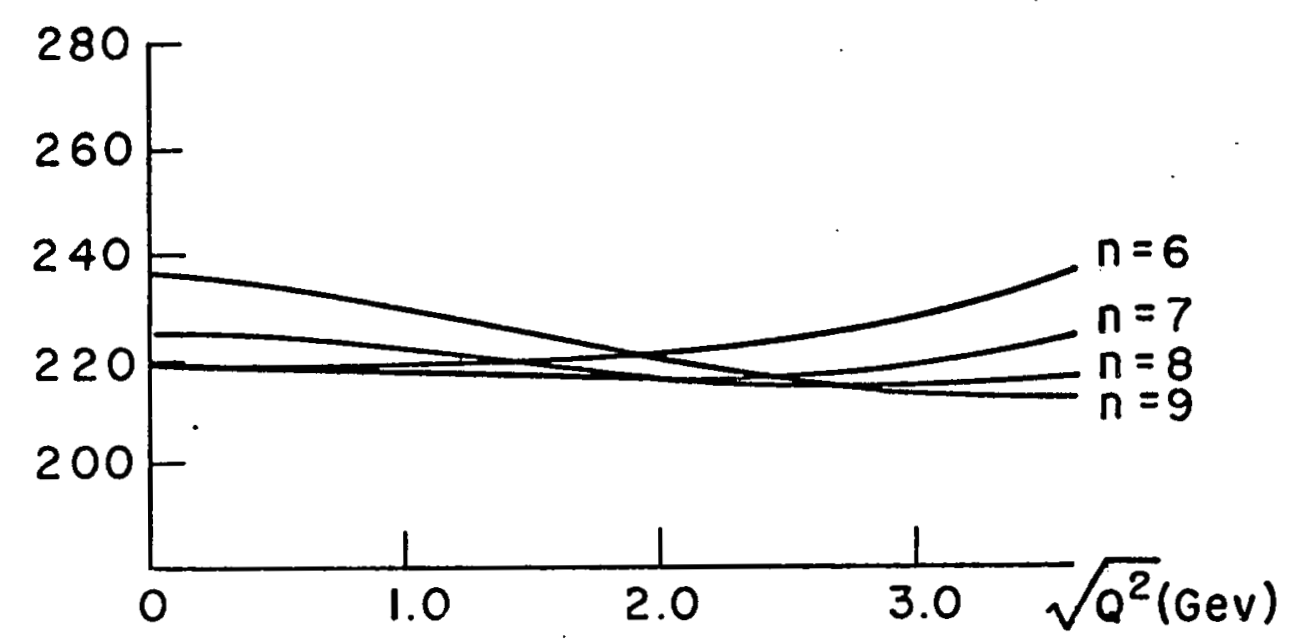

Fig 4-(a)

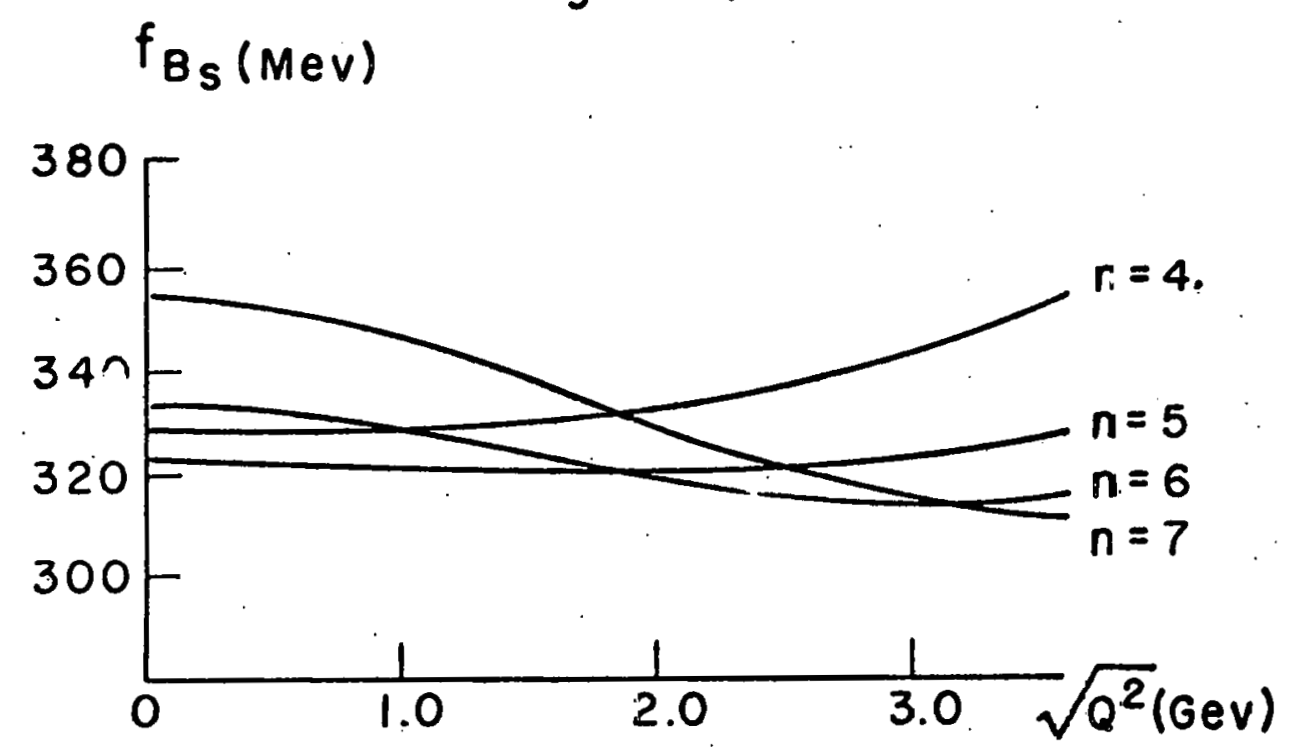

Fig 3-(b)

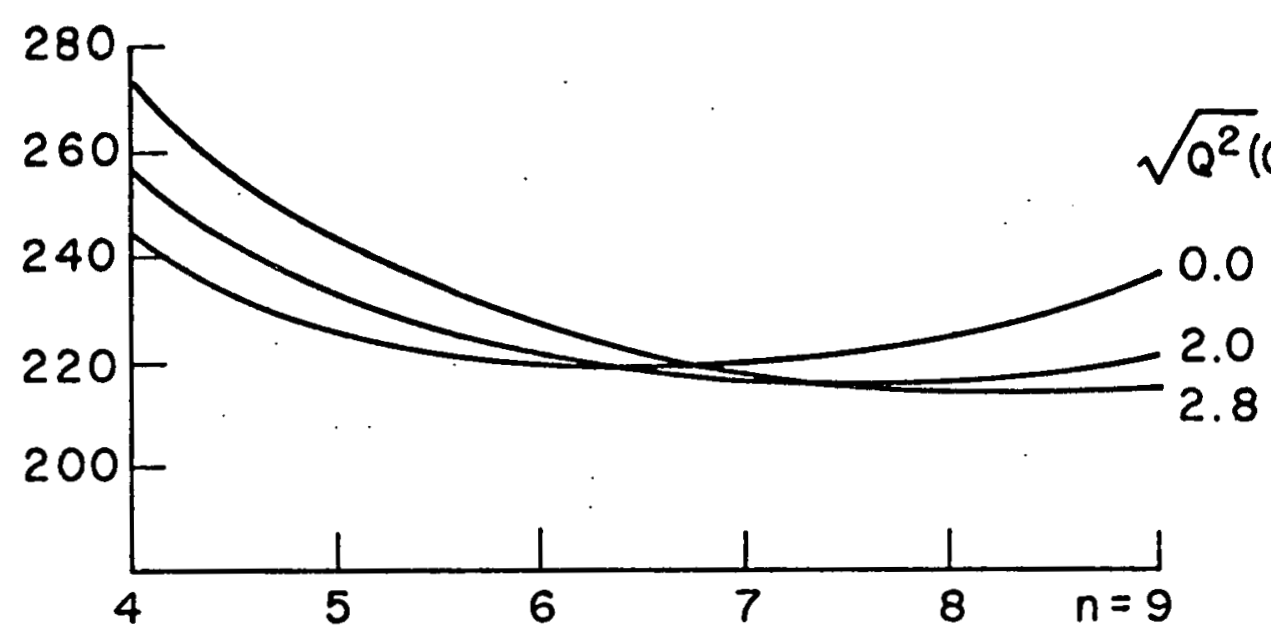

Fig $4-(b)$

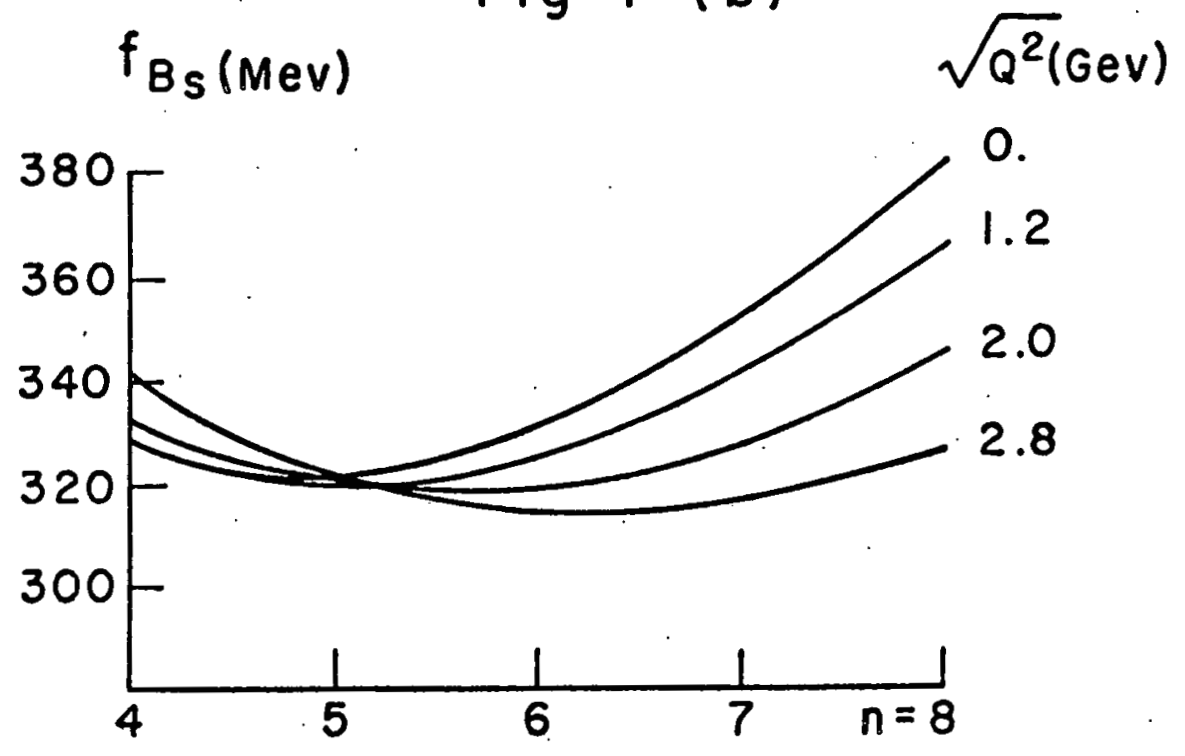


Fig $5-(a)$

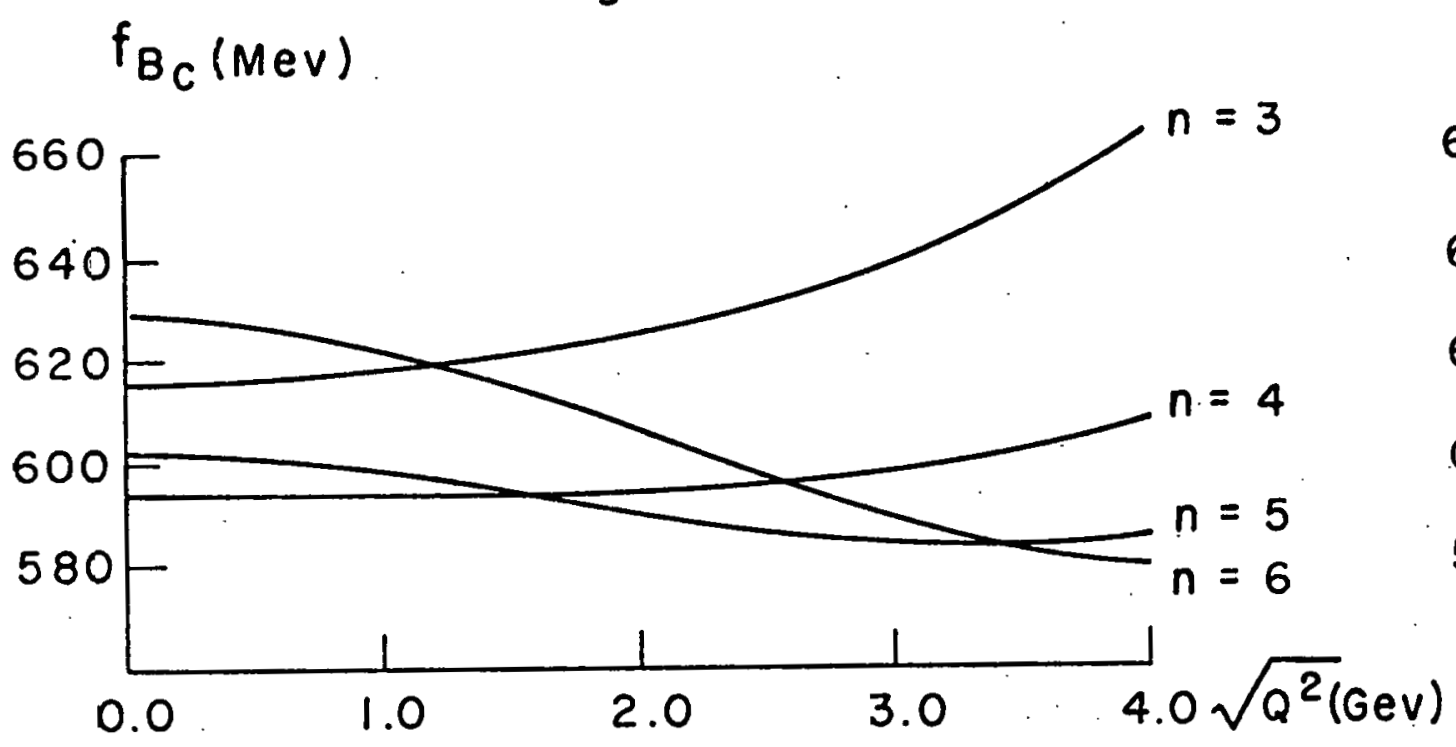

Fig 5-(b)

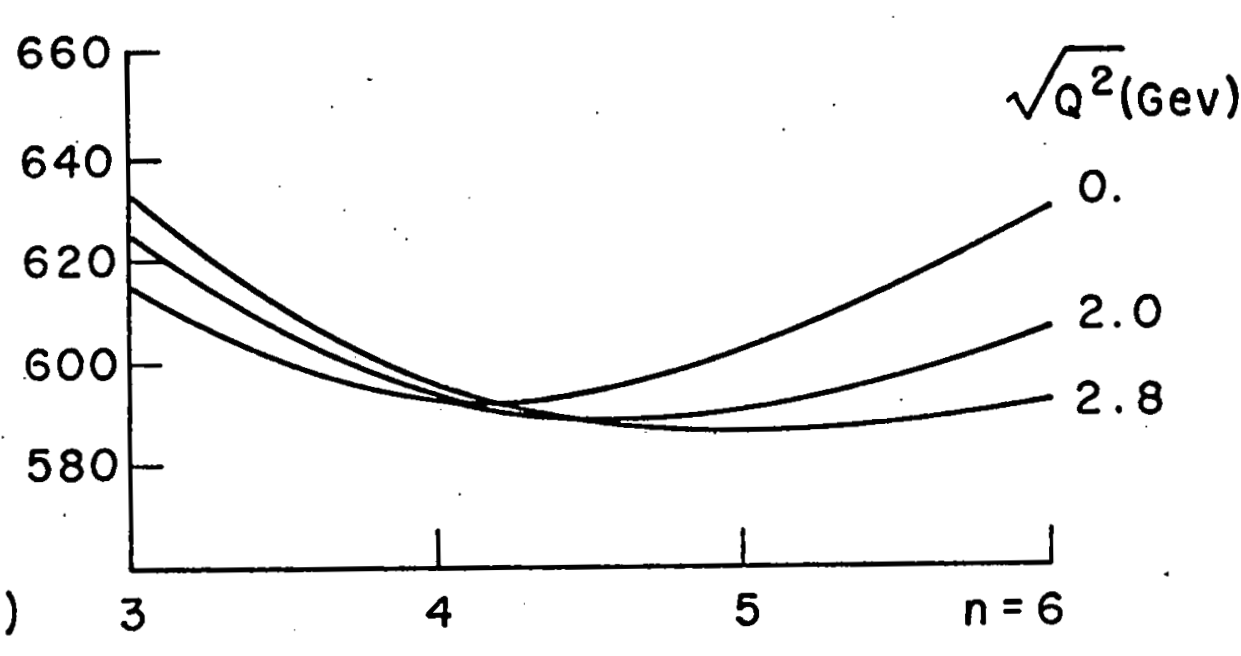

